\title{
Evaluation of Circle Diameter by Distributed Tactile Information in Active Tracing
}

\author{
Hiroyuki Nakamoto, Futoshi Kobayashi, and Fumio Kojima \\ Graduate School of System Informatics, Kobe University, Kobe 657-8501, Japan \\ Correspondence should be addressed to Hiroyuki Nakamoto; hiroyuki.nakamoto@kojimalab.com
}

Received 15 March 2013; Accepted 14 May 2013

Academic Editor: Aiguo Song

Copyright ( 2013 Hiroyuki Nakamoto et al. This is an open access article distributed under the Creative Commons Attribution License, which permits unrestricted use, distribution, and reproduction in any medium, provided the original work is properly cited.

Active touch with voluntary movement on the surface of an object is important for human to obtain the local and detailed features on it. In addition, the active touch is considered to enhance the human spatial resolution. In order to improve dexterity performance of multifinger robotic hands, it is necessary to study an active touch method for robotic hands. In this paper, first, we define four requirements of a tactile sensor for active touch and design a distributed tactile sensor model, which can measure a distribution of compressive deformation. Second, we suggest a measurement process with the sensor model, a synthesis method of distributed deformations. In the experiments, a five-finger robotic hand with tactile sensors traces on the surface of cylindrical objects and evaluates the diameters. We confirm that the hand can obtain more information of the diameters by tracing the finger.

\section{Introduction}

Human voluntarily touches an object and perceives its characteristic features. The procedure is called as active touch. Gibson has indicated that the active touch is important for shape perception [1]. In addition, it has been reported that two-position recognition threshold on human finger tip is 2 to $3 \mathrm{~mm}$. The threshold gives us an impression of large value. Then, Loomis and collins have reported that the sensitivity for stimulus in active touch becomes high [2]. In order to perceive shape of objects, the results indicate an importance of moving hands. Therefore, not only a development of tactile sensor but also a measurement method based on exploratory moving is necessary for developing dexterous robotic hand.

Various studies of robotic hands with tactile sensor have been discussed [3-7]. The tactile sensors are used for recognition. Okamura and Cutkosky have proposed a method which detects small textural features by tracing with robotic fingers [4]. In the case, the hand recognized a local area on an unknown object, and it is called the local exploratory operation. Pribadi et al. have proposed a method to estimate the contour of unknown objects [5]. Allen and Michelman have focused on sensing of global object shapes and fitting shapes to object models [6]. Wang and Li have applied a tactile sensor for surface tracking of a manipulator [7]. Nakamoto et al. have proposed a method of classifying the shape of an object that a five-finger robot is grasping [8]. The purposes of them are recognitions of unknown area by robotic hands with tactile sensor. Using tactile information, Wang and Li have proposed a feedback to control a robotic arm interacting with soft human skin [9] and have applied an end-effector with a tactile sensor to a massaging work [10]. These studies indicate the importance and effectiveness of robotic hands with tactile sensor. Therefore, the robotic hands are required to increase their applications and become more dexterous.

In order to become more dexterous, the robotic hands should obtain much information of an object. The information is classified roughly into two groups: global rough information and local detail information. The related studies described previously recognize unknown objects and obtain global information. On the other hand, active touch is a method to obtain detailed and local information of objects. If robotic hands can obtain detailed information by active touch as necessary, the information can be used for realizing dexterous operations. The discussions of active touch for 


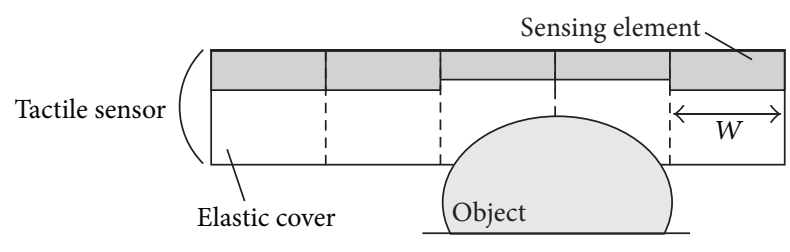

Figure 1: Cross-section view of sensor model.

robotic hands are not still enough, and requirements of the tactile sensor which can obtain detailed information by active touch should be also discussed.

In this paper, we aimed at recognizing details of the target area by robotic hand with tactile sensors. The purpose of this study realizes higher spatial resolution than structural spatial resolution of the sensor by tracing the area (hereafter in this paper the tracing operation is referred to as the "active tracing"). In the following sections, we define four requirements of a tactile sensor for active tracing and design a distributed tactile sensor model, which can measure a distribution of compressive deformation. Next, we suggest a measurement process by the sensor model. Then, we confirm the method that can obtain combined distribution to distinguish the circular diameter by robotic hand. Finally, we discuss these results and make a conclusion.

\section{Active Touch by Distributed Tactile Sensor}

2.1. Sensor Model. In this section, we describe a modeling of distributed tactile sensor based on knowledge about human skin structure and reveal a mechanism of the high-resolution sensing with active tracing.

Human touches the environment through the skin which covers all of the body. The skin is soft and has a lot of receptors. The receptors detect stimuli to the skin. The skin over the body is one organ which is not being divided along the positions of receptors. Then, we extract the following four features of skin:

(i) softness;

(ii) distributed receptors;

(iii) sheet-like structure;

(iv) output based on touch area and depth.

A 2D model of distributed tactile sensor based on the first three of four features is shown in Figure 1. The model has a structure which distributes sensor elements and is covered with an elastomer over the elements. The width of one sensor element is $W$, and there is no gap between the elements. The seamless elastomer deforms along the shape of touched object. Each sensor element outputs value which responds to the deformation of the upper elastomer. In this model, the width-direction spatial resolution is decided by the width of the sensor element. Therefore, the spatial resolution of the model is $W$, and the two-position recognition threshold of the sensor is larger than $W$.
2.2. State Transition in Active Tracing. When the model touches an object with a constant depth, the contact width between the model and the object is $L$. The maximum number of sensor elements, which have outputs based on the contact, is $\lceil L / W\rceil+1$. The width of output distribution is also $\lceil L / W\rceil+$ 1. Because the elemental width $W$ is the surplus width about the contact position, the strict position relationship between the object and the element is not determined uniquely from the output distribution. Therefore, even if the number of the output elements $\lceil L / W\rceil+1$ and the deformation are constant, the output distribution has changing possibilities within the width $W$ due to the contact position. In general, the width $W$ and the differences of the output distribution correspond to the spatial resolution and errors of the sensor, respectively. They are designed and selected according to applications of the sensor. In this study, the active tracing uses the width $W$ and propose a sensing method which traces the sensor within the width $W$.

The contact state transition is shown in the next example. Figure 2 shows an example which traces three times in increments of $W / 4$. The number of measurement is four. In this time, the object is fixed to the environment, and there is no friction between the object and the sensor. $t$ means the time step of the trace. The contact width is less than $2 W$ at $t=0$. The object contacts the elastomer of $e_{3}$ and $e_{4}$ sensor elements. Then, only $e_{3}$ and $e_{4}$ sensor elements output values of the deformation. At $t=1$, the sensor traces $W / 4$ rightward, the deformation occurs on the elastomer of $e_{2}$ element. The deformations of $e_{3}$ and $e_{4}$ become large and small, respectively. Next, at $t=2$, the deformations of $e_{2}$ and $e_{3}$ become large and that of $e_{4}$ becomes small. Finally, at $t=3$, the deformation of $e_{2}$ becomes large, those of $e_{3}$ and $e_{4}$ become small. In this process, even if the contact width is constant, there are the differences of deformation by tracing smaller width than the spatial resolution.

2.3. Combination of Sensor Outputs. In this section, we describe a combination method of sensor outputs in active tracing. The width of one tracing is $W / N$, which is based on the element width $W$. $N$ means the number of measurement in one active tracing. In Figure 2, the element $e_{2}$ has no deformation at $t=0$, but the deformation becomes large as the tracing progresses. On the other hand, the deformation of the element $e_{4}$ becomes progressively smaller. These are continuous changes along the time steps of the trace, and we define the outputs of the sensor as $e_{i}(j)(i=1, \ldots, E, j=$ $0, \ldots, N-1)$ where $E$ is the number of the sensor element, and then, we can put them in order of $\left(e_{i}(j)\right)_{j=0,1, \ldots, N-1}=$ $e_{i}(0), e_{i}(1), \ldots, e_{i}(N-1)$. The order is decided by the trace direction.

Next, we focus on the relationship of the elements. The output of element $e_{i+1}$ at $t=0$ corresponds to that of $e_{i}$ at $t=N$. Assuming that the sensor elements have the same characteristics about output, we can consider that the output $e_{i+1}(0)$ of element $e_{i+1}$ at $t=0$ and the output $e_{i}(N-1)$ of element $e_{i}$ at $t=N-1$ are continuous change between the elements by tracing in the width $W / N$. The combination of distributed outputs is obtained by putting in order of 
TABLE 1: Width of voltage distribution (number of nonzero volt element).

\begin{tabular}{lcccccc}
\hline Number of measurement & $\phi 10$ & $\phi 15$ & $\phi 20$ & $\phi 25$ & $\phi 30$ & $\phi 35$ \\
\hline$N=1$ & 3 & 4 & 4 & 5 & 5 & 5 \\
$N=2$ & 9 & 9 & 9 & 10 & 10 & 11 \\
$N=4$ & 17 & 17 & 18 & 18 & 19 & 11 \\
$N=8$ & 34 & 36 & 37 & 39 & 41 & 20 \\
\hline
\end{tabular}

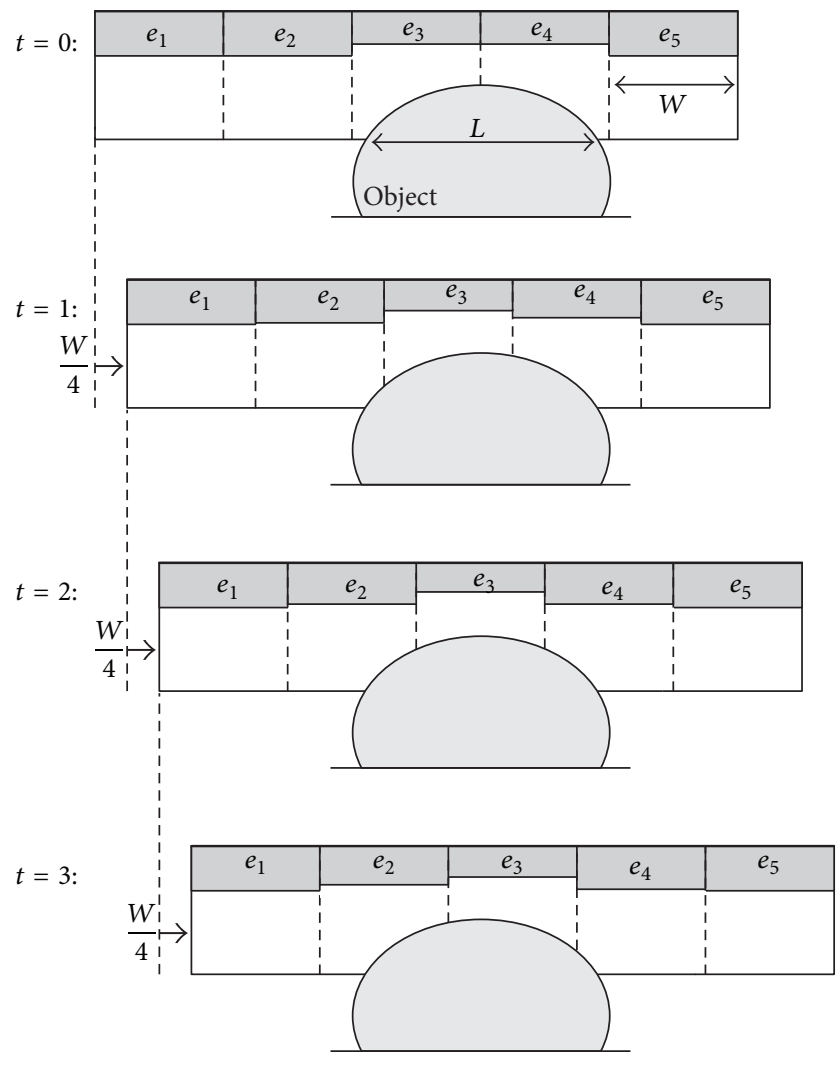

FIGURE 2: Principle of active tracing.

$\left(e_{1}(j)\right)_{j=0,1, \ldots, N-1},\left(e_{2}(j)\right)_{j=0,1, \ldots, N-1}, \ldots,\left(e_{E}(j)\right)_{j=0,1, \ldots, N-1}$. The combined distribution is the continuous changes by tracing in the width $W / N$ from $t=0$ to $N-1$ and includes the details of the contact deformation.

\section{Tactile Sensor for Robotic Hand}

3.1. Distributed Tactile Sensor. In order to verify the effectiveness of combined outputs of the distributed tactile sensor in active tracing, we designed and made the sensor as shown in Figure 3. Although the measurement principle of the sensor uses a resistance change at the time of the compressive deformation of the pressure-sensitive conductive rubber as well as the sensor which Shimojo [11], Nakamoto et al. [8], and others proposed as a tactile sensor for a multifinger robot hand, the structure of the sensor is designed in consideration of the features which were described in Section 2.1. The sensor has a three-layer structure. It consists of an elastomer

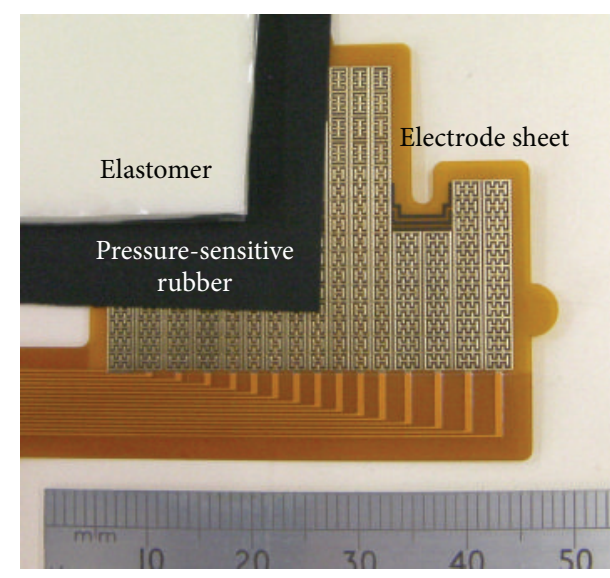

FIGURE 3: Prototype sensor.

sheet, a pressure-sensitive conductive rubber, and an electrode substrate. The elastomer sheet is $2 \mathrm{~mm}$ in thickness. If the sheet is too hard, it cannot deform along the contact surface. In contrast, if it is too soft, the pressure on the surface is absorbed by the sheet and the pressure-sensitive rubber cannot deform by the surface pressure. Then, Young's modulus of the elastomer sheet is selected as the same value of the pressure-sensitive rubber. The pressure-sensitive rubber, which is $0.5 \mathrm{~mm}$ in thickness, has a characteristic which the electric resistance changes by compressive deformation. The electrode sheet is a flexible substrate. The electrode pattern on the substrate is shown in Figure 4. The electrode is used for the measurement of electric resistance of the pressure-sensitive rubber. An electrode (hereafter is called a unit electrode) is $2 \mathrm{~mm}$ square. The size includes $0.2 \mathrm{~mm}$ gaps between neighborhood electrodes. Each unit electrode consists of two electrodes for applying voltage and receiving current. The applying-voltage electrodes are printed on the surface of the substrate, and the receiving electrodes are wired inside the substrate via throughholes. The numbers of applying-voltage and receiving electrodes are 16 in the vertical and horizontal directions in Figure 3, respectively. In order to be designed for fitting on the robotic finger, the arrangement of unit electrodes is designed like Figure 3. The number of unit electrodes in the substrate is 204 . We also made a multiplexer circuit which selects unit electrodes and amplifies output voltages. A FPGA (Field-Programmable Gate Array) on the same circuit synchronizes selection and measurement. The sampling time of the 204 units is $1 \mathrm{~ms}$.

3.2. Fundamental Evaluation of Prototype. We made experiments to evaluate the relationship of outputs of the prototype 


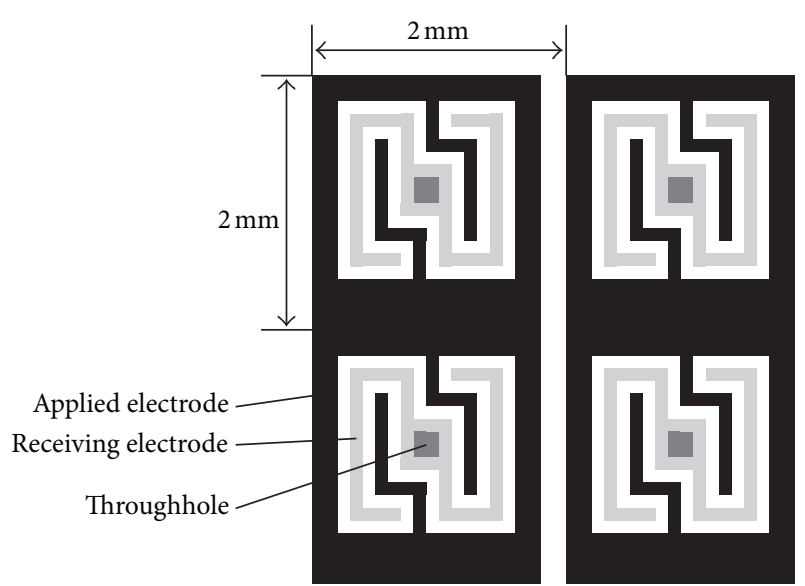

Figure 4: Design of electrode.
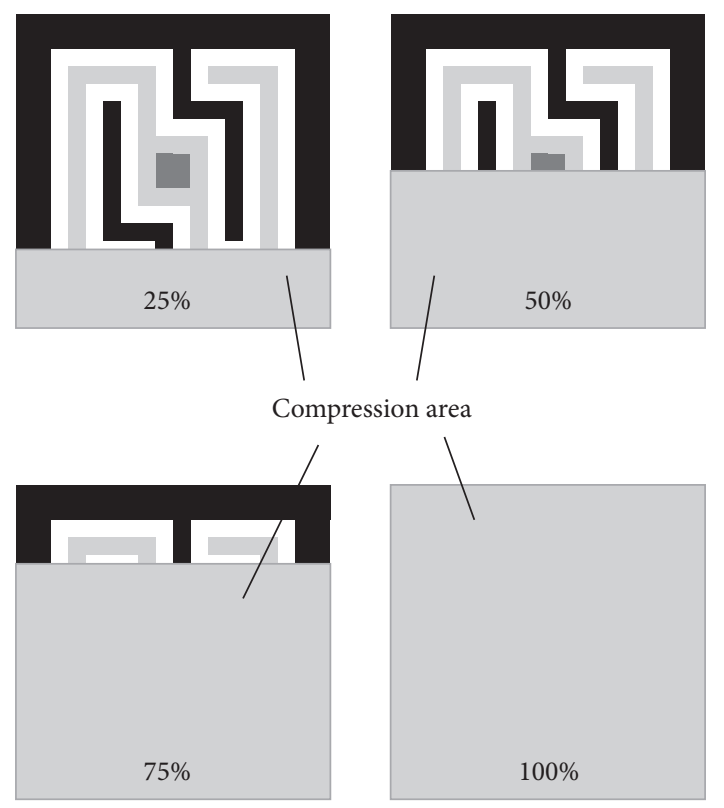

FIGURE 5: Conditions of compression experiment.

sensor to pressured areas. The relationship corresponds to the forth feature in Section 2.1. In the experiments, we used a 2-axis motorized stage to deform the sensor accurately. The contact object was an acrylic cube. The cube pressed all or partial areas of a unit electrode, and then, we evaluate outputs of the sensor. Then, we decided four different contact conditions that the pressed areas of a unit electrode are 100, 75,50 , or $25 \%$ as shown in Figure 5. The cubic surface was set accurately parallel to the sensor surface, and the motorized stages move the cube in the direction of compression. The range of the movement was from 0 to $1.0 \mathrm{~mm}$, and the length of one movement was $0.1 \mathrm{~mm}$.

The exprimental results are shown in Figure 6. The horizontal and vertical axes in Figure 6 are the press depth and output voltage, respectively. The results show that the output voltage became high by a constant slope as the amount of compression becomes large from 0 to $1.0 \mathrm{~mm}$. The ratios

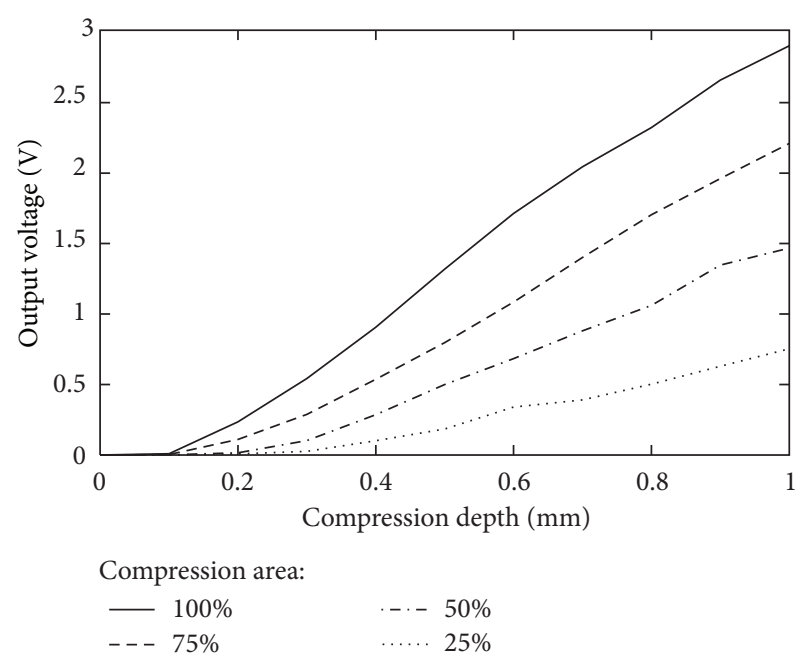

FIGURE 6: Compression depth versus output voltage relation.

of compression area correspond to the output voltages in especially $1.0 \mathrm{~mm}$. For example, about 4 times of the output voltage of $25 \%$ area were mostly in agreement with the output voltage of $100 \%$ area. However, when compressive deformations were small, there were also the results that the output voltages had not changed. For example, the output voltage of $50 \%$ area increased after $0.2 \mathrm{~mm}$. This cause is considered that small compressive deformations were absorbed by the surface elastomer sheet. As described previously, the contact area of compressive deformation is proportional to the output voltage in the condition of a certain amount of press depth. An equation of the relation is defined later by the least mean square approximation,

$$
v_{o}=3.45 p q-0.39
$$

where $v_{o}$ is the output voltage, $p(0 \leq p \leq 1.0)$ and $q$ are the contact area and the compression depth, respectively. Since the amount of deformation equals the product of the contact area and the compression depth, the output voltage is proportional to the amount of deformation. Furthermore, if the deformation of the surface is a partial area, the output voltage increases in response to the area. Therefore, we confirmed that the prototype has the fourth function described in Section 2.1.

\section{Experiments with Active Tracing}

4.1. Multifinger Robotic Hand. A photograph of multifinger robotic hand and the arrangement of its DOFs are shown in Figure 7 [12]. The robotic hand has five fingers and a total of 16 degrees of freedom and has tactile sensors shown in Figure 7 on its finger pads. Although the tactile sensor also covers finger tip and side, the finger contacts only with the finger pad in the next experiments. The length between the middle finger tip and the palm bottom is $290 \mathrm{~mm}$. The hand is controlled by a PC with RT Linux as the operating system. It can precisely be operated by the PD control. 


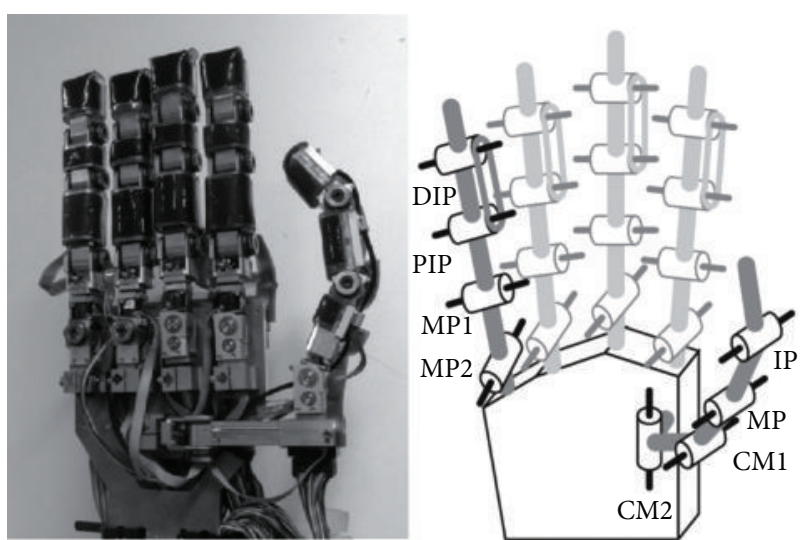

FIgURE 7: Multifinger robotic hand.

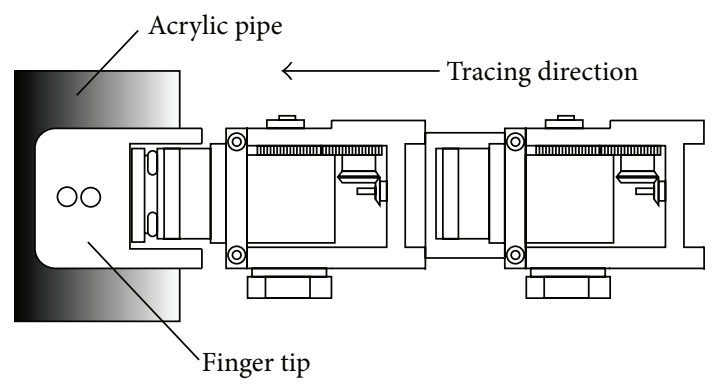

FIgURE 8: Tracing direction.

4.2. Combination of Output Voltage. We used seven acrylic pipes as objects. The robotic hand touches and traces on them in experiments. The pipes are 10, 15, 20, 25, 30, 35, and $40 \mathrm{~mm}$ in diameter. The lengths of the pipes are $20 \mathrm{~mm}$. First, the pipe is fixed on the table. Next, the finger presses the pipe $0.7 \mathrm{~mm}$ in the normal direction to the pipe. Then, the finger traces on the pipe in the direction perpendicular to the shaft of the pipes and the direction parallel to the surface of the sensor. The finger tip is equipped with a 3-axis force sensor and kept contact force constant. Because the unit electrode is 2 -mm-squre, we decided $1.0,0.5$, and $0.25 \mathrm{~mm}$ as the lengths of one tracing. In response, the finger traced and measured 2, 4, and 8 times, respectively. The tracing direction is shown in Figure 8. The finger can trace on the pipes at a constant velocity and measured output distributions at each tracing length. After measurements of each 2, 4, and 8 times were completed, we obtained combined distributions from the measured outputs by the order described in Section 2.3. Because outputs in the direction of the shaft do not change by using the pipes as objects, we extracted only output distributions of a pipe-radial direction that had the highest output value. In order to compare results, we also obtained outputs without tracing.

The results are shown in Figure 9. In order to compare easily, outputs of zero volt part except for both ends of the distribution are not shown in Figure 9. $N$ expresses the number of measurement in one active tracing as described in Section 2.3. In the graphs, the horizontal axes mean the sequential element number of combined voltage distribution, and the vertical axes mean the output voltage of each element. Figure 9(a) shows the results of one measurement without tracing, and the output distributions are most of the same shapes and the widths even if the diameters of pipe are different. The widths of valued distribution are from 3 to 5 , and they are not useful to distinguish the pipe diameters. It is difficult to estimate the diameter of the pipe from an output of single measurement. On the other hand, the combined outputs in $N=2$ and $N=4$ measurements have double and quadruple widths of single-measurement distributions, respectively. The width of output distribution tends to increase as the pipe diameter becomes large. The distribution widths are from 34 to 44 in $N=8$ and depend on the pipe diameters.

Next, we focus on the shapes of output distribution. The shapes of distribution in $N=2$ are almost the same shapes of distribution expanded to double singlemeasurement outputs in the width direction. In contrast, the combined distributions in $N=4$ have some high values and peaks of arc shape. There are differences between the shapes of $N=4$ and those of the quadruple distribution of single measurement even though the pipe diameters are the same. Then, the shapes of distribution have difference by increasing the number of measurement. The results show that the combined distribution has details of the shape by active tracing. According to Figure 9(d) which includes the results in $N=8$, the distribution widths of each diameter have difference. The loose peaks of distribution are obtained by tracing the finger in $N=4$ and $N=8$. We extend our investigations of the distribution widths and the loose peaks.

We extracted feature quantities from the combined distributions. Table 1 shows the widths of the combined distributions. The widths mean the numbers of element of distribution not including 0 volt. Table 2 shows the averages of absolute difference between neighborhood elements. Each distribution width has correspondence with value which is obtained by multiplying the number of measurement by the width in $N=1$. Then, the variation of distribution widths is 3 under the conditions of $N=1$ and $N=2$. In $N=4$, the variation is 4 and is 7 at $N=8$. The results in $N=8$ show that the finger can classify diameters of pipe by the width of combined distribution and some thresholds. The combined distributions reflect small change of compressive deformation and give high spatial resolution to the sensor. On the other hand, we can confirm the resolution in the height direction of distribution in Table 2. The averages become smaller along with the increasing of the number of measurement. Then, the distributions in Figure 9(d) have gentler curves than those in Figure 9(a). Furthermore, the large diameter pipes have the small averages. For example, in $N=4$ or $N=8$, each average becomes small gradually as the pipe diameters become large. A large diameter pipe has a gentle curve on a touch surface, and the averages reflect the details despite the small deformations. The increasing of the number of measurement strengthens the tendency to that. Therefore, we confirmed that the spatial resolution of the sensor becomes high by the tracing measurement.

In order to confirm the spatial resolution for pipe diameter classification with the sensor tracing, we focused on 
TABLE 2: Average of difference between neighborhood members of voltage distribution.

\begin{tabular}{lccccccc}
\hline Number of measurement & $\phi 10$ & $\phi 15$ & $\phi 20$ & $\phi 25$ & $\phi 30$ & $\phi 35$ & 0.454 \\
\hline$N=1$ & 0.522 & 0.481 & 0.436 & 0.413 & 0.485 & 0.418 \\
$N=2$ & 0.321 & 0.320 & 0.299 & 0.309 & 0.293 & 0.289 & 0.275 \\
$N=4$ & 0.181 & 0.177 & 0.169 & 0.159 & 0.140 & 0.141 & 0.140 \\
$N=8$ & 0.103 & 0.097 & 0.095 & 0.089 & 0.089 & 0.083 & 0.079 \\
\hline
\end{tabular}

(The unit is V.)

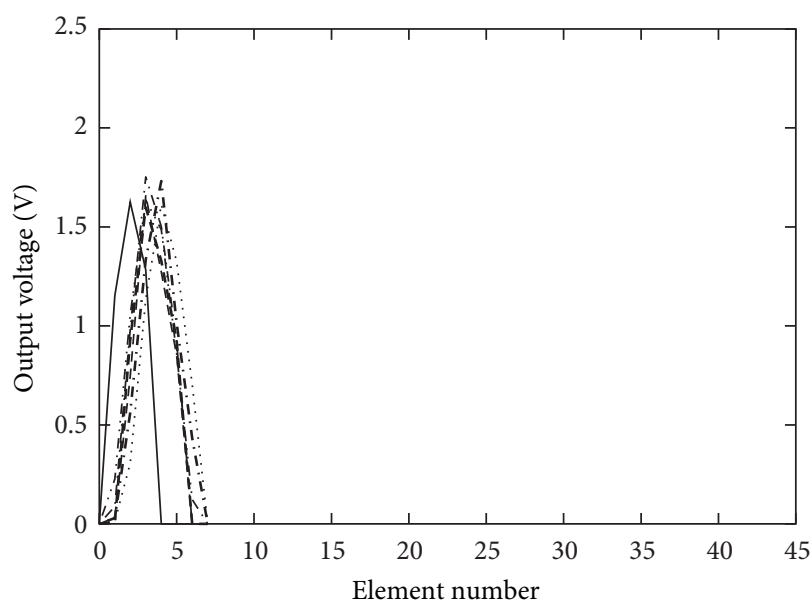

(a) $N=1$

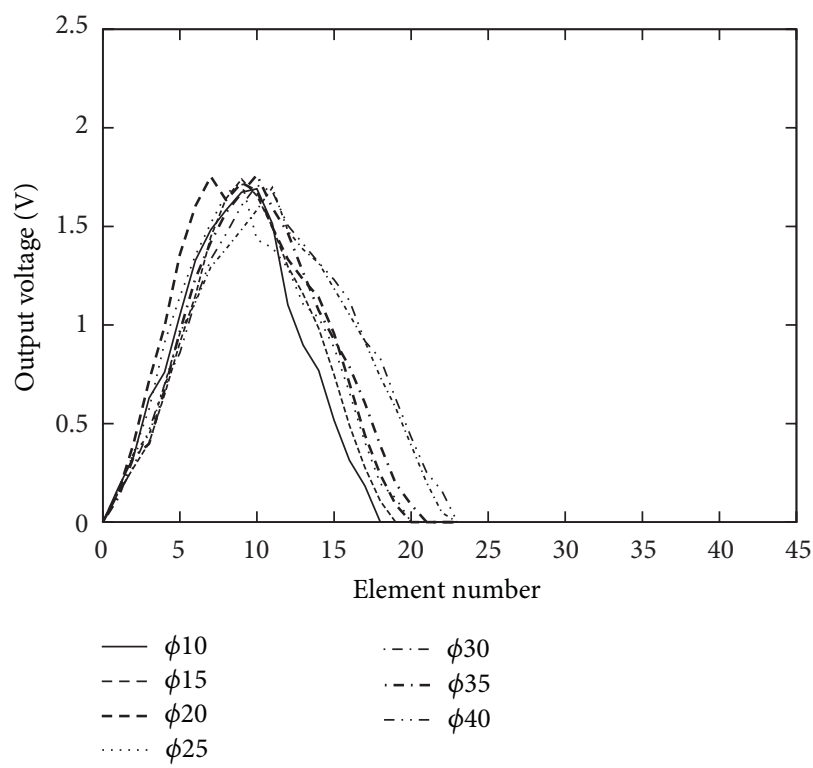

(c) $N=4$

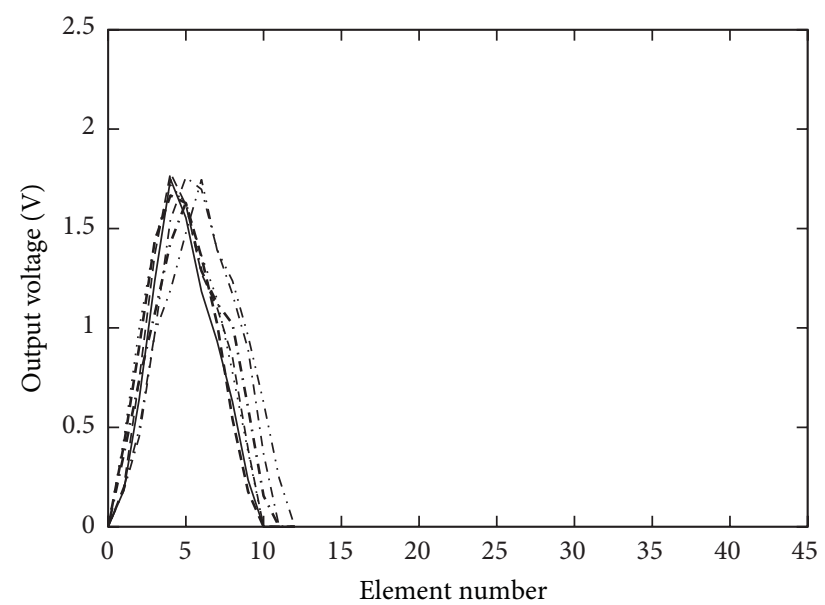

(b) $N=2$

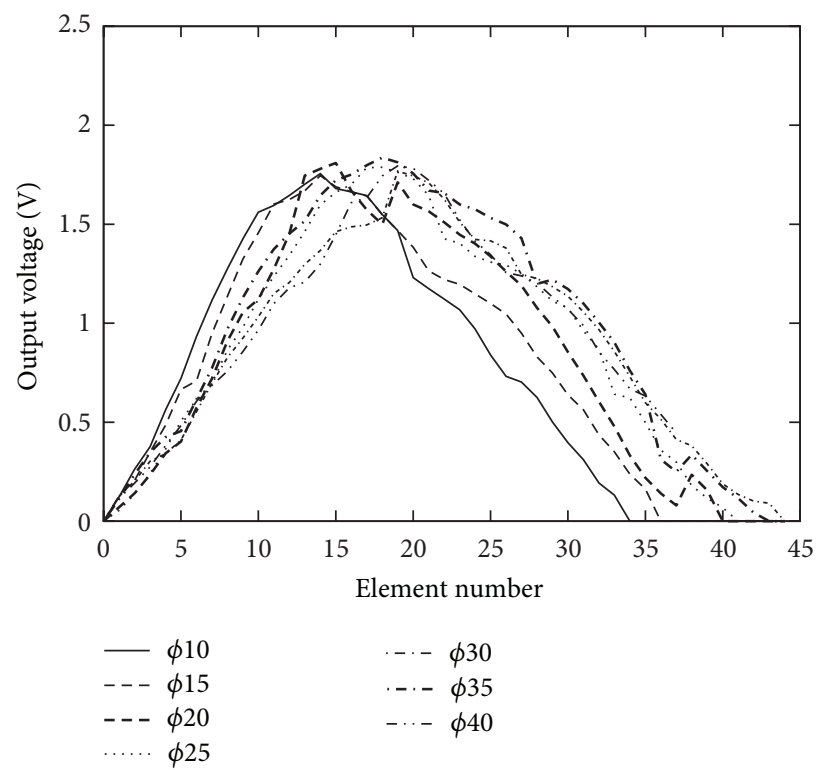

(d) $N=8$

FIGURE 9: Results of combined voltage distribution.

the classification by the widths of combined distribution. In Table 1, the distributions in $N=1$ and $N=2$ have three widths. However, we described in Section 2.2 that a width of combined distribution is not constant because of effects of relative position between the sensor and the object. For example, in the case of $N=1$, the distribution width of $25 \mathrm{~mm}$ pipe in diameter is 4 or 5 . Through repetitive experiments, the single measurement could distinguish 10 or $40 \mathrm{~mm}$ in diameter by the distribution width without depending on the relative position. The result means that the spatial resolution of the sensor was $30 \mathrm{~mm}$ in diameter. On the other hand, the measurements of 4 times had the spatial resolution of $15 \mathrm{~mm}$ in diameter. The resolution of the measurement of 8 times was $10 \mathrm{~mm}$ in diameter. These results 
show that the robotic hand can distinguish $10 \mathrm{~mm}$ in pipe diameter. We confirmed that the robotic hand can distinguish the diameters at the high resolution by tracing the finger.

\section{Conclusion}

In this paper, in order to obtain details of local area of an object, we decided the conditions of distributed tactile sensors and verified the effectiveness of tracing the finger with the tactile sensor. As the results of the experiment by the robotic finger, the finger could obtain combined distributions and distinguish pipe diameters by $10 \mathrm{~mm}$. Although we have used 1D distributions for combined distributions in this paper, the tactile sensors on the robotic hand can output $2 \mathrm{D}$ voltage distributions. In order to obtain combined $2 \mathrm{D}$ distributions, we are extending the proposed method and are trying to obtain $3 \mathrm{D}$ touch surfaces from combined $2 \mathrm{D}$ distributions at the next step of this study.

The sensor can obtain details of local area by the proposed method. Although the miniaturization of the tactile sensor is required in order to improve the accuracy of touch perception, we also consider that it is necessary to combine the proposed local area measurement and a global area measurement [13]. To increase efficiency of the measurement, a high-speed performance is required of the global area measurement. We will apply the sensor to a robotic hand and propose a measurement method of whole shape of an object by the combination of the local and global area measurement.

\section{References}

[1] J. J. Gibson, "Observations on active touch," Psychological Review, vol. 69, no. 6, pp. 477-491, 1962.

[2] J. M. Loomis and C. C. Collins, "Sensitivity to shifts of a point stimulus: an instance of tactile hyperacuity," Perception and Psychophysics, vol. 24, no. 6, pp. 487-492, 1978.

[3] T. Mouri, H. Kawasaki, and K. Umebayashi, "Developments of new anthropomorphic robot hand and its master slave system," in Proceedings of the IEEE IRS/RSJ International Conference on Intelligent Robots and Systems (IROS '05), pp. 3474-3479, August 2005.

[4] A. M. Okamura and M. R. Cutkosky, "Feature detection for haptic exploration with robotic fingers," International Journal of Robotics Research, vol. 20, no. 12, pp. 925-938, 2001.

[5] K. Pribadi, J. S. Bay, and H. Hemami, "Exploration and dynamic shape estimation by a robotic probe," IEEE Transactions on Systems, Man and Cybernetics, vol. 19, no. 4, pp. 840-846, 1989.

[6] P. K. Allen and P. Michelman, "Acquisition and interpretation of 3-D sensor data from touch," IEEE Transactions on Robotics and Automation, vol. 6, no. 4, pp. 397-404, 1990.

[7] J. Wang and Y. Li, "Surface-tracking of a 5-DOF manipulator equipped with tactile sensors," in Proceedings of the 11th International Conference on Control, Automation, Robotics and Vision (ICARCV '10), pp. 2448-2453, Singapore, December 2010.

[8] H. Nakamoto, F. Kobayashi, N. Imamura, H. Shirasawa, and F. Kojima, "Shape classification in rotation manipulation by universal robot hand," in Proceedings of the IEEE/RSJ International Conference on Intelligent Robots and Systems (IROS '08), pp. 5358, Nice, France, September 2008.
[9] J. Wang and Y. Li, "A study on a robotic arm contacting with human skin using tactile sensing feedback strategies," in Proceedings of the IEEE International Conference on Robotics and Biomimetics (ROBIO '10), pp. 1559-1564, Tianjin, China, December 2010.

[10] J. Wang and Y. Li, "Massaging human feet by a redundant manipulator equipped with a tactile sensor," in Proceedings of the IEEE/ASME International Conference on Advanced Intelligent Mechatronics (AIM '10), pp. 7-12, Montreal, Canada, July 2010.

[11] M. Shimojo, "Mechanical filtering effect of elastic cover for tactile sensor," IEEE Transactions on Robotics and Automation, vol. 13, no. 1, pp. 128-132, 1997.

[12] W. Fukui, F. Kobayashi, F. Kojima et al., "Fingertip force and position control using force sensor and tactile sensor for universal robot hand II," in Proceedings of the Workshop on Robotic Intelligence in Informationally Structured Space (RIISS '11), pp. 43-48, Paris, France, April 2011.

[13] R. L. Klatzky and S. J. Lederman, "Stages of manual exploration in haptic object identification," Perception and Psychophysics, vol. 52, no. 6, pp. 661-670, 1992. 

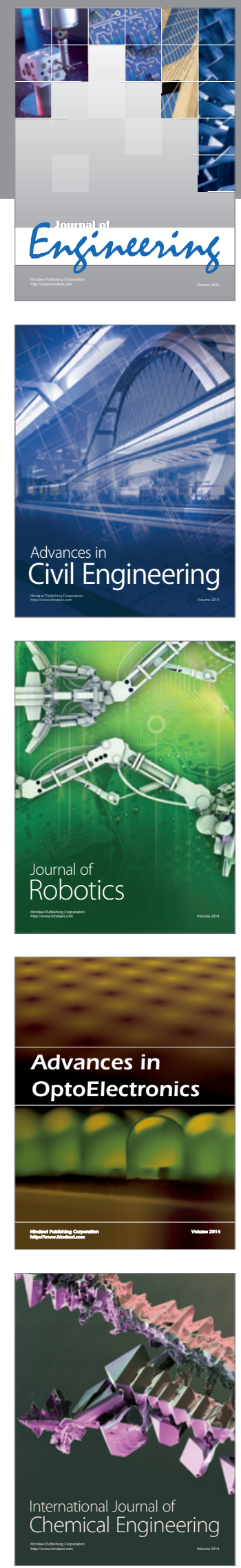

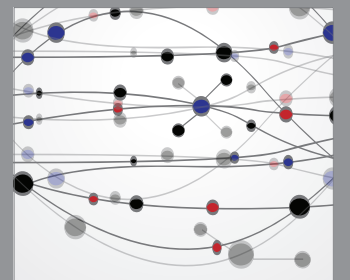

The Scientific World Journal
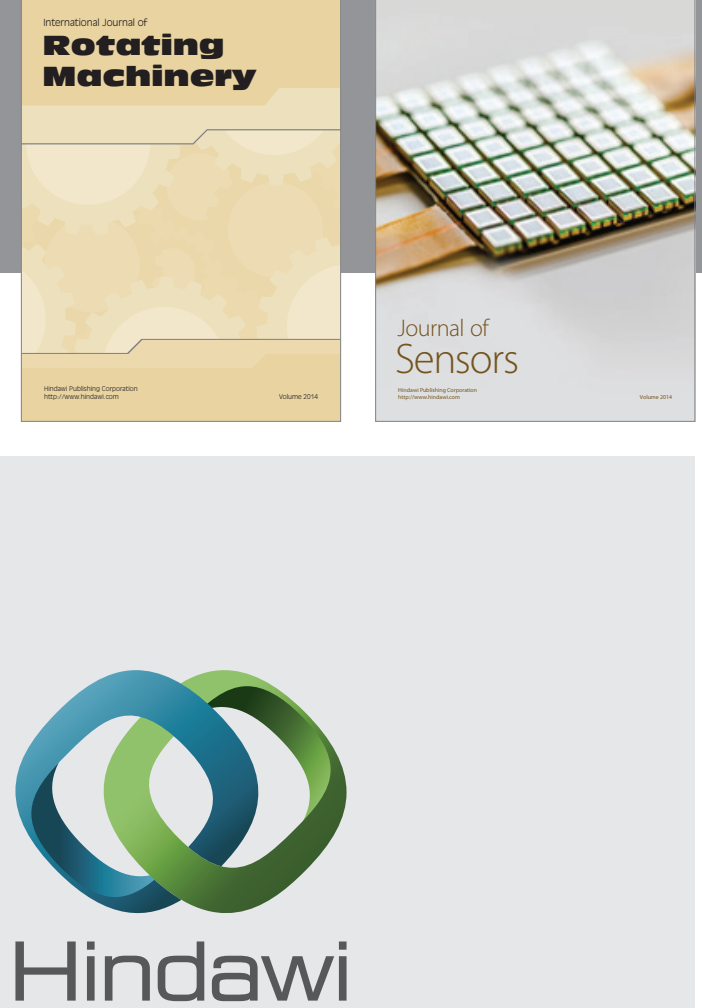

Submit your manuscripts at http://www.hindawi.com
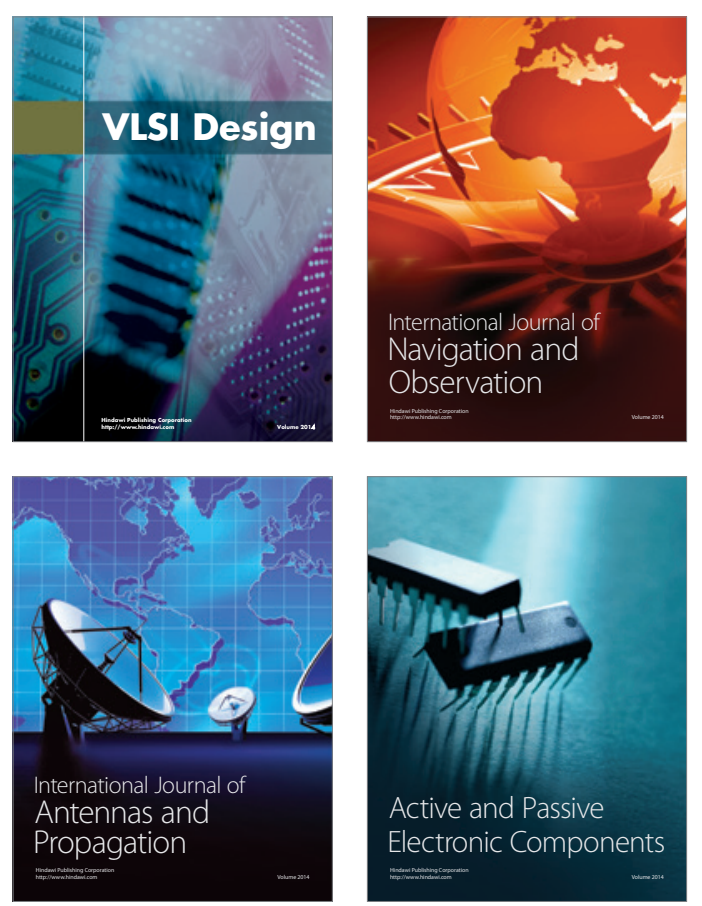
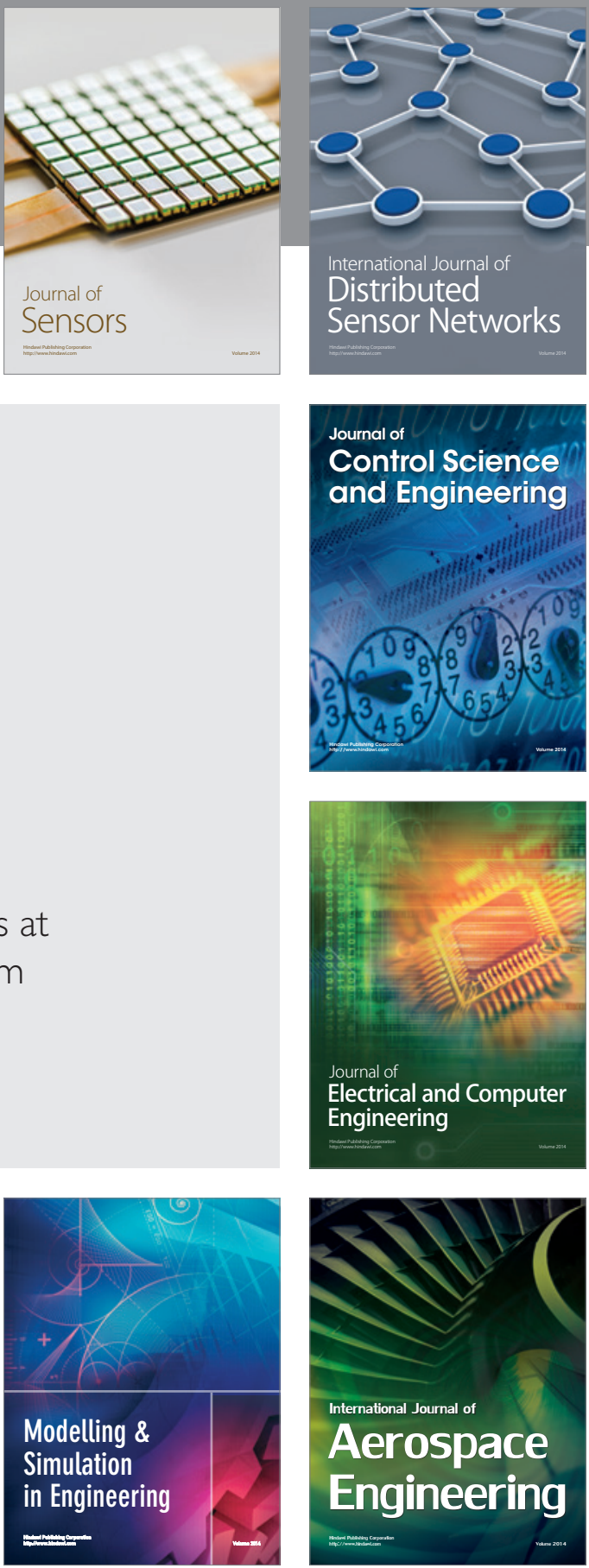

Journal of

Control Science

and Engineering
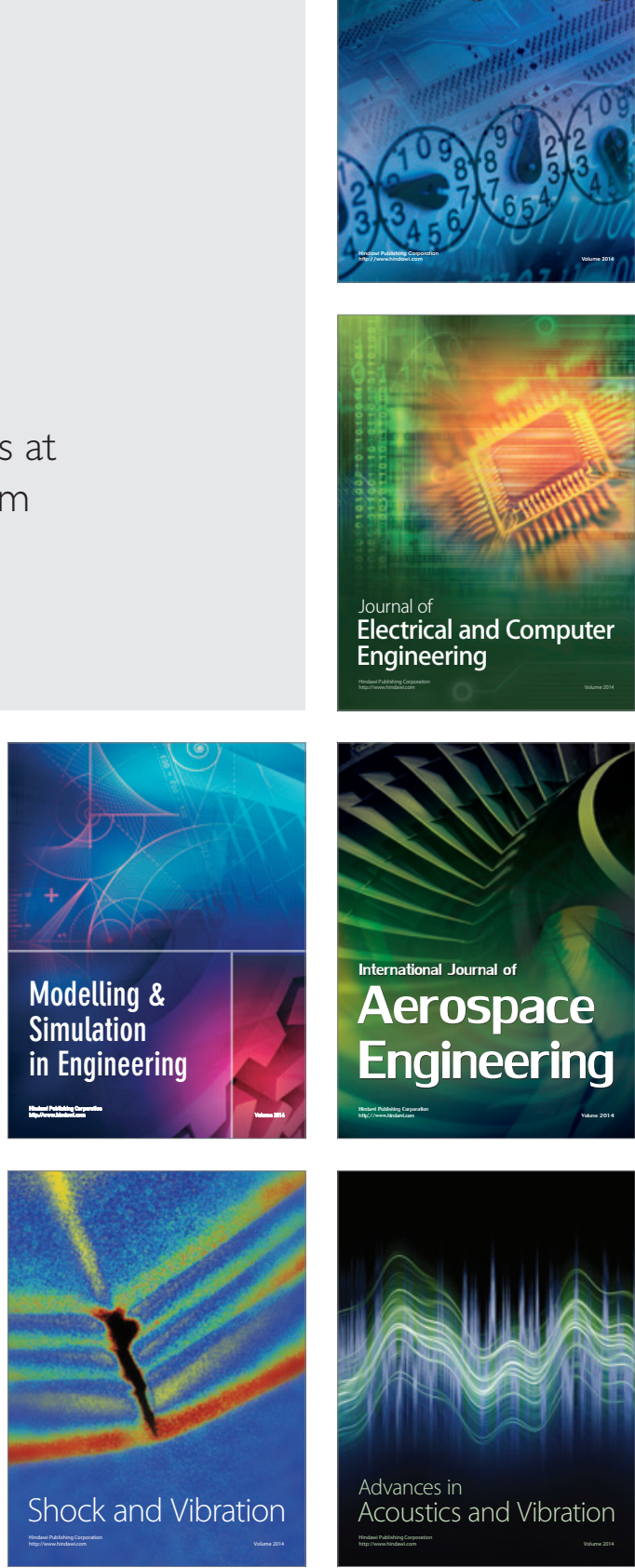